\title{
Unstructured Grid Solutions for Incompressible Laminar Flow over a Circular Cylinder Using a Particular Finite Volume-Finite Element Method
}

\author{
Mahdi Yousefifard, Parviz Ghadimi, and Rahim Zamanian \\ Department of Marine Technology, Amirkabir University of Technology, Hafez Avenue No. 424, P.O. Box 15875-4413, Tehran, Iran \\ Correspondence should be addressed to Parviz Ghadimi; pghadimi@aut.ac.ir
}

Received 12 October 2012; Revised 25 February 2013; Accepted 25 February 2013

Academic Editor: Sergio Nardini

Copyright (C) 2013 Mahdi Yousefifard et al. This is an open access article distributed under the Creative Commons Attribution License, which permits unrestricted use, distribution, and reproduction in any medium, provided the original work is properly cited.

\begin{abstract}
A numerical modeling of a 2D Navier-Stokes equation by a particular vertex centered control volume framework on an unstructured grid is presented in this paper. Triangular elements are applied with an effective high performance fully coupled algorithm, to simulate incompressible laminar flow over a circular cylinder. The cell face velocities in the discretization of the continuity and momentum equations are calculated by a combined linear and momentum interpolation scheme, respectively, and their performances are compared. Flow analyses have been conducted based on various Reynolds numbers up to 200 for the steady and unsteady flows using structured and unstructured grids. The robustness and accuracy of the scheme in the unstructured mesh are proved using the benchmark problems of incompressible laminar flow over a circular cylinder at low and medium Reynolds numbers. Results have been compared with the structured grid results, both cases with equal cell numbers and same strategy for the mesh refinement. Current results display good agreement with the experimental values. Overall, it is shown that, using the suggested method for the current problem, unstructured grids are highly competitive with the structured grids.
\end{abstract}

\section{Introduction}

Unstructured grids have been used in order to model objects with complicated geometry in different areas of hydrodynamics. This type of grid has also been used for simulation of the flows over bluff bodies. The efficiency and numerical accuracy of unstructured mesh have been compared with those of structured grids on test cases of fundamental fluid flow by many researchers. Flow over a circular cylinder is considered as one of the fundamental fluid mechanics problems. The flow field over the cylinder is symmetric at low values of Reynolds number. As the Reynolds number increases, flow begins to separate behind the cylinder causing vortex shedding which is an unsteady phenomenon. Flow around circular bodies has a majority of past research. Many engineering problems can be simulated by this simplification. Flow around circular particles in Lagrangian method is an example of these problems. Using unstructured grid for the solution of flow in a complicated geometry could be the best remedy.

Early studies of flow around a circular cylinder have been conducted at low Reynolds number with structured grid. Researchers such as Bloor [1], Roshko [2], and Tritton [3] focused on the flow in the near wake region of the cylinder. Experimental work on the pressure and velocity distribution on circular cylinder flow between Reynolds numbers of 10 to 80 was carried out by Nishioka and Sato [4]. The rapid development of CFD has made a wide range of investigations possible. Satisfactory results have been obtained between Reynolds numbers of 100 to 300 from CFD simulation $[5,6]$. In recent years, employing unstructured meshes in CFD applications has been growing fast, which makes it necessary to evaluate the precision and robustness of this scheme. Zhang et al. [7] made a comparison of structured and unstructured grid solutions for flow over a circular cylinder. 


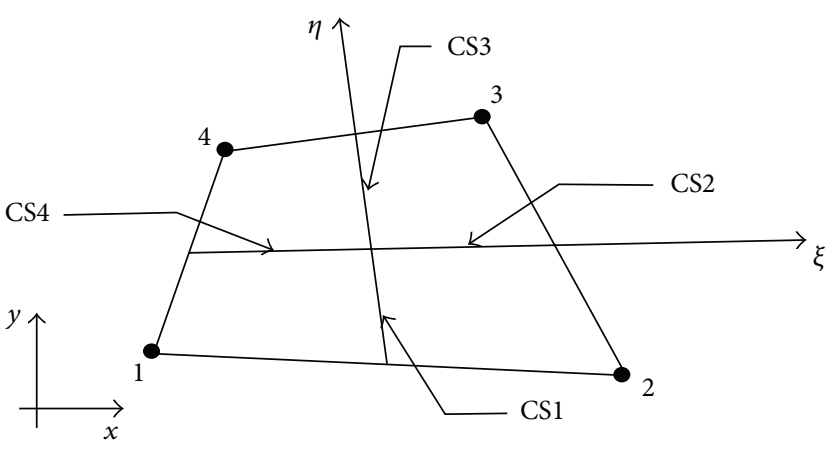

(a)

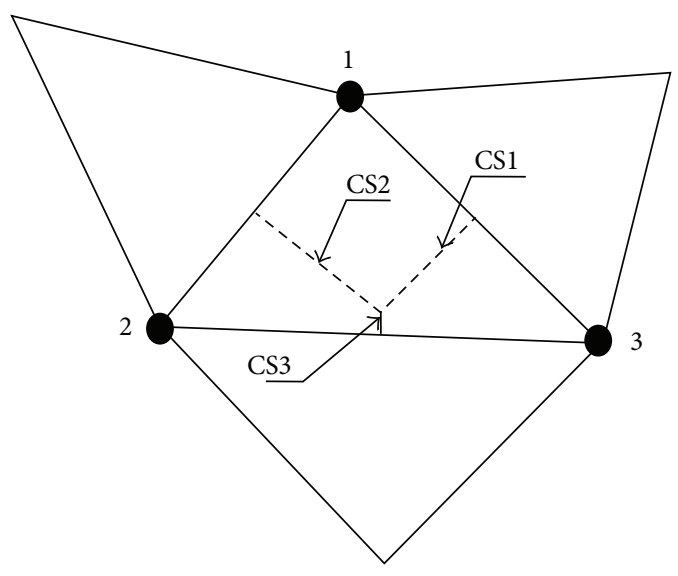

(b)

FIGURE 1: Element and integration points on control surfaces: (a) quadrilateral element and (b) tetrahedral element.

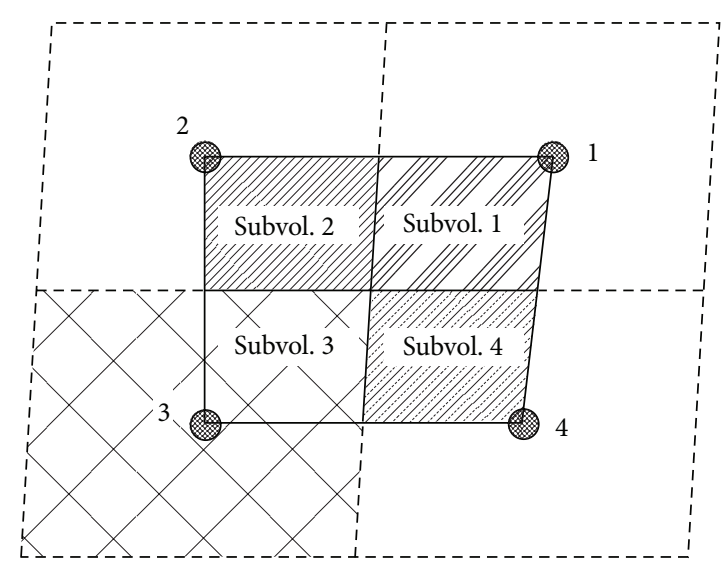

(a)

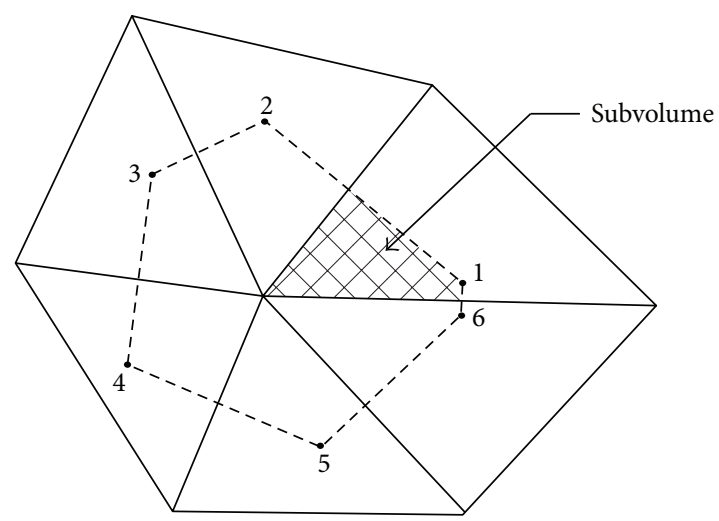

(b)

FIGURE 2: Subvolumes of elements: (a) quadrilateral element and (b) tetrahedral element.

TABLE 1: Comparison of value of the recirculation length and separation angle for $\mathrm{Re}=10,20$, and 40 .

\begin{tabular}{lccc}
\hline $\begin{array}{l}\text { Reynolds } \\
\text { number }\end{array}$ & Source & $L_{\text {sep }}$ & $\theta_{\text {sep }}$ \\
\hline \multirow{4}{*}{10} & Ding et al. [9] & 0.252 & 30 \\
& Present (unstructured grid) & 0.260 & 29.5 \\
& Present (structured grid) & 0.262 & 29.6 \\
\hline \multirow{4}{*}{20} & Ding et al. [9] & 0.93 & 44.1 \\
& Present (unstructured grid) & 0.911 & 43.8 \\
& Present (structured grid) & 0.907 & 43.8 \\
\hline \multirow{4}{*}{40} & Ding et al. [9] & 2.20 & 53.5 \\
& Present (unstructured grid) & 2.19 & 54.2 \\
& Present (structured grid) & 2.19 & 54.1 \\
\hline
\end{tabular}

Also, Lysenko [8] used unstructured meshes for the solution of flow over a number of bluff bodies.
Navier-Stokes equations in their velocity-pressure formulation for a $2 \mathrm{D}$ incompressible viscous flow are applied in a tetrahedral grid system. Fully coupled algorithm based on Rhie and Chow [10] scheme could be a remedy to suppress the checkerboard problem. In this paper, a collocated grid has been used in the vertex centered control volume framework. In the mass equation, velocity components on the control volume surfaces are defined according to Alisadeghi and Karimian [11] formulation, and simple linear approach is used for momentum equations [12]. High performance collocated scheme based finite element-finite volume method has been developed for the rectangular grid and tested on flow over a circular cylinder. This code is deployed for the triangular grid used for the simulation of flow in the same geometry. The accuracy and discretization method for the rectangular and triangular grids are the same. The proposed scheme, by using Karimian and Wang technique [11, 12], achieves highly accurate results in a short time. Needless to say, using this combination leads to simplicity of scheme and more efficiency for the unstructured grids. 


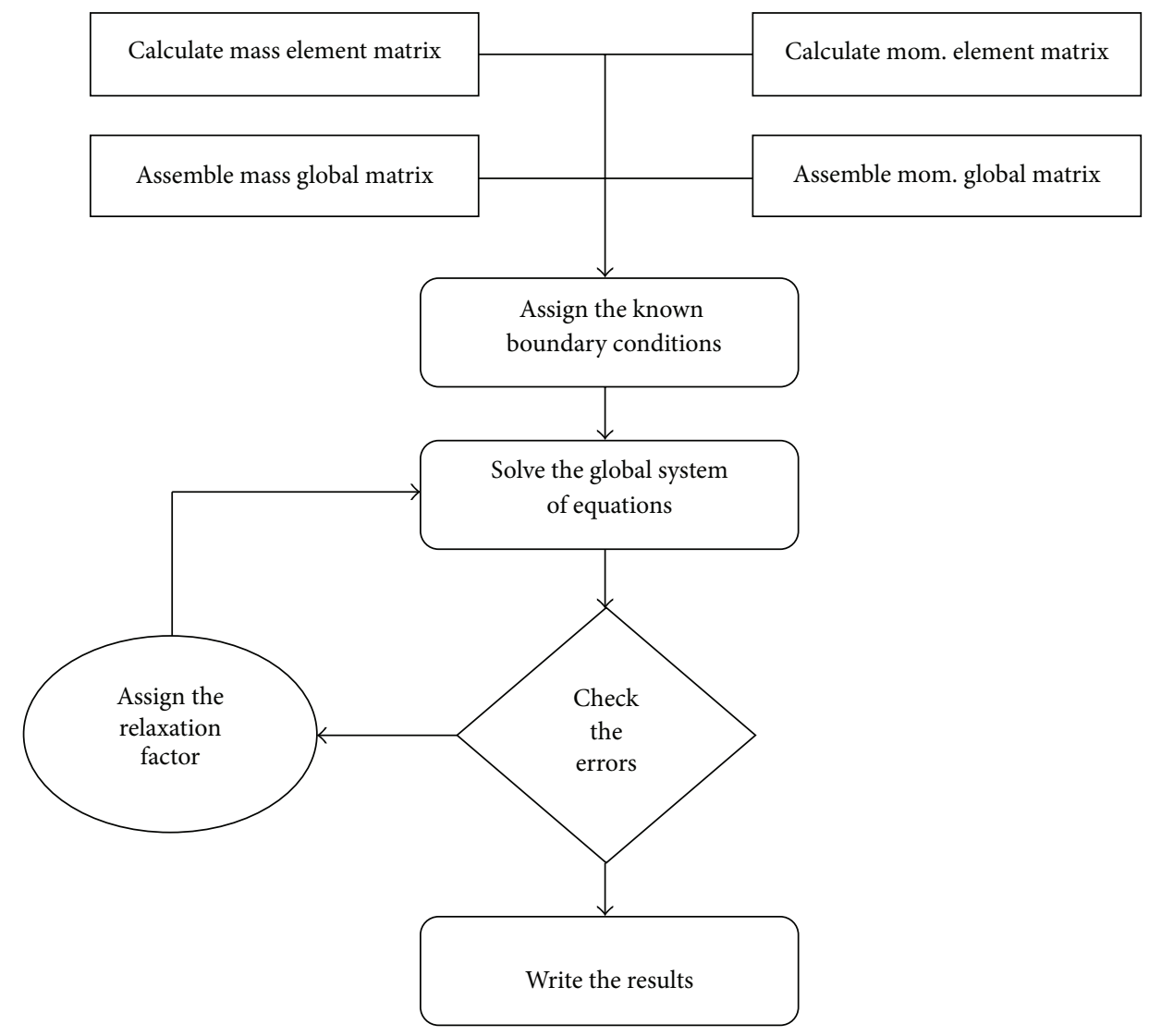

FIGURE 3: Solution procedure.



FIgURE 4: Configuration of the test case.

\section{Governing Equations}

Formulation for the two-dimensional incompressible Navier-

Stokes is given by

$$
\begin{gathered}
\frac{\partial u}{\partial t}+u \frac{\partial u}{\partial x}+v \frac{\partial u}{\partial y}=-\frac{\partial p}{\partial x}+\frac{1}{\operatorname{Re}}\left(\frac{\partial^{2} u}{\partial x^{2}}+\frac{\partial^{2} u}{\partial y^{2}}\right), \\
\frac{\partial v}{\partial t}+u \frac{\partial v}{\partial x}+v \frac{\partial v}{\partial y}=-\frac{\partial p}{\partial x}+\frac{1}{\operatorname{Re}}\left(\frac{\partial^{2} v}{\partial x^{2}}+\frac{\partial^{2} v}{\partial y^{2}}\right), \\
\frac{\partial u}{\partial x}+\frac{\partial v}{\partial y}=0
\end{gathered}
$$

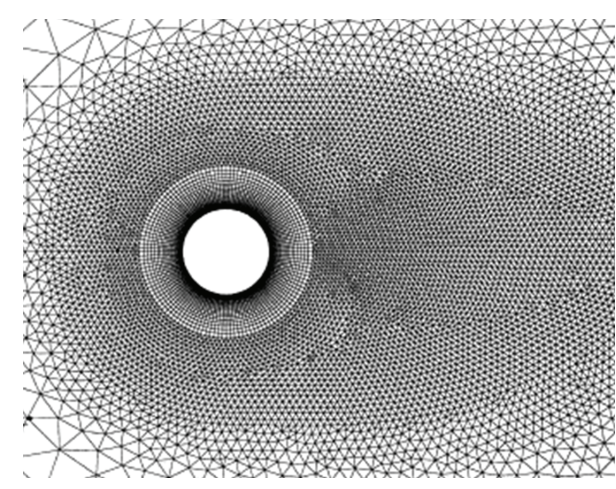

FIGURE 5: Unstructured triangular grids around the cylinder.

where Reynolds number is defined as $\operatorname{Re}=\rho U_{\infty} L_{\infty} / \mu$ and $\rho$ is the density and $\mu$ is the viscosity of the fluid. Equation (1) in conservation form, integrated in a control volume $\forall$, gives

$$
\begin{aligned}
\int_{\forall} \frac{\partial u}{\partial t} d \forall= & -\int_{\forall} \frac{\partial p}{\partial x} d \forall \\
& +\int_{\forall}\left[\frac{1}{\operatorname{Re}}\left(\frac{\partial^{2} u}{\partial x^{2}}+\frac{\partial^{2} u}{\partial y^{2}}\right)-\frac{\partial^{2} u}{\partial x}-\frac{\partial u v}{\partial y}\right] d \forall,
\end{aligned}
$$




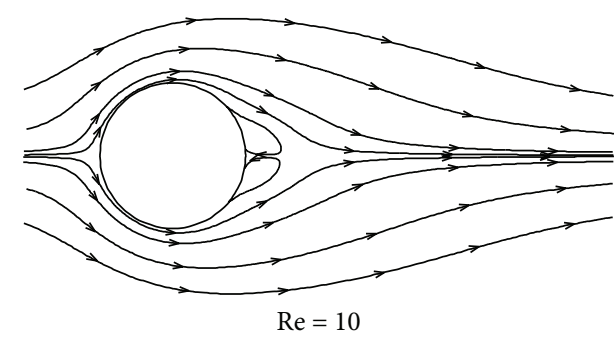

(a)



(b)



(c)

Figure 6: Streamline contour for $\operatorname{Re}=10,20$, and 40.

$$
\begin{aligned}
\int_{\forall} \frac{\partial u}{\partial t} d \forall= & -\int_{\forall} \frac{\partial p}{\partial y} d \forall \\
& +\int_{\forall}\left[\frac{1}{\operatorname{Re}}\left(\frac{\partial^{2} v}{\partial x^{2}}+\frac{\partial^{2} v}{\partial y^{2}}\right)-\frac{\partial u v}{\partial x}-\frac{\partial^{2} v}{\partial y}\right] d \forall \\
& \int_{\forall}\left(\frac{\partial u}{\partial x}+\frac{\partial v}{\partial y}\right) d \forall=0 .
\end{aligned}
$$

Rewriting (2) and applying the Green's Theorem result in

$$
\begin{aligned}
\int_{\forall} \frac{\partial u}{\partial t} d \forall= & -\int_{\forall} \frac{\partial p}{\partial x} d \forall \\
& +\oint_{S}\left(\frac{1}{\operatorname{Re}} \frac{\partial u}{\partial x}-u^{2}\right) d y-\left(\frac{1}{\operatorname{Re}} \frac{\partial u}{\partial y}-u v\right) d x \\
\int_{\forall} \frac{\partial u}{\partial t} d \forall= & -\int_{\forall} \frac{\partial p}{\partial y} d \forall \\
& +\oint_{S}\left(\frac{1}{\operatorname{Re}} \frac{\partial v}{\partial x}-u v\right) d y-\left(\frac{1}{\operatorname{Re}} \frac{\partial v}{\partial y}-v^{2}\right) d x \\
& \int_{\forall}\left(\frac{\partial u}{\partial x}+\frac{\partial v}{\partial y}\right) d \forall=0 .
\end{aligned}
$$

Momentum interpolation should be always used to calculate the cell face velocity in the discretized continuity equation to prevent the checkerboard pressure field [12]. The improved scheme of Alisadeghi and Karimian [11] is implemented to evaluate the momentum interpolation of velocities at the cellfaces. According to this scheme, momentum interpolation of velocity in conservation of mass equation is given by

$$
\widehat{u}_{i p}=U_{\text {ave }}+\frac{1}{d}\left(-\frac{\partial p}{\partial x}+\rho\left(u \frac{\partial v}{\partial y}-v \frac{\partial u}{\partial y}\right)\right),
$$

where $d=\rho \bar{q}_{i p} / \Delta S_{u p}+\mu / l^{2}$ and $l$ is a suitable diffusion length scale for the element. Velocity components in the momentum equations are found by linear interpolation. All cell face velocities of the control volumes are calculated by the combination of momentum and linear interpolation methods for mass and momentum equations. For the mass equation, momentum interpolation is used, and for the momentum equation, linear interpolation is implemented.

Unstructured tetrahedral element is used to test the algorithm. Figure 1 shows the general form of this element and subcontrol volumes. In this method, the control volume is centered around the vertices and the cells are divided into subcontrol volumes that make up the main control volume. The nodes store the average variable values over the control volumes. Integration point is on the midpoint of each control surface. Elements are divided into three quadrilateral subcontrol volumes. These subcontrol volumes are shown in Figure 2.

\section{Solution Procedure}

An iterative scheme is used in the solution of the NavierStokes equations for the structured and unstructured grids. Continuity and momentum equations are solved simultaneously. At first, the initial data except the boundary values of the known functions are applied. Subsequently, an iterative procedure is implemented as shown in Figure 3.

At the end of each iteration, a simple relaxation method is used to damp the oscillations in the obtained results which 


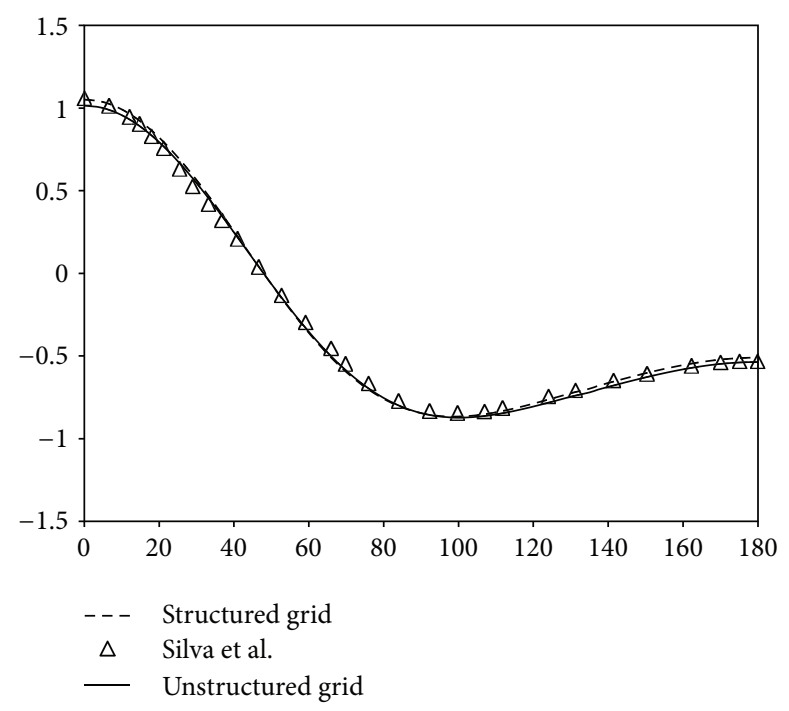

Figure 7: Pressure coefficient distribution, between $(\theta=0)$ and $(\theta=180): \operatorname{Re}=20$.

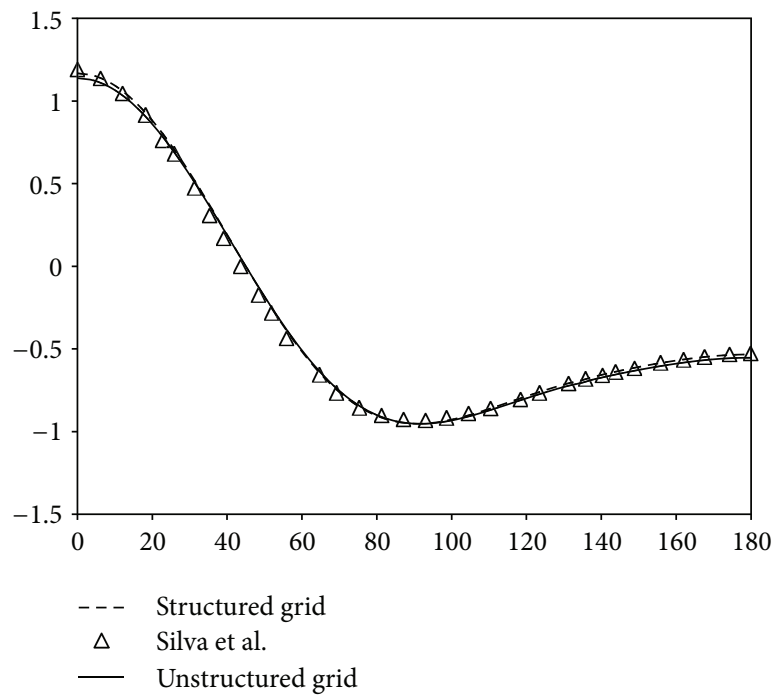

Figure 8: Pressure coefficient distribution, between $(\theta=0)$ and $(\theta=180): \operatorname{Re}=40$.

increases speed of the convergence. This procedure is used for each time step and continued until the defined maximum time is reached.

\section{Flow past a Circular Cylinder}

The capabilities of the proposed method for the unstructured grids are demonstrated by simulating the laminar flow over a circular cylinder. The flow around a circular cylinder at different Reynolds numbers $\left(\operatorname{Re}=U_{\infty} D / \nu\right)$ is computed to study the influence of several parameters, where $U_{\infty}$ is the free-stream velocity, $D$ is the cylinder diameter, and $v$ is the kinematic viscosity.

The domain geometry is indicated in Figure 4 . To minimize the effects of the upstream and downstream boundaries on the flow, these boundaries are located at distances sufficiently away from the cylinder. For all computations, these distances are $L_{u}=10 D$ and $L_{D}=50 D$, respectively, which are measured from the center of the cylinder. In the present study, the domain has a width $H=10 D$ and the blockage $(B=D / H)$ is 0.1 . These dimensions were chosen in order to minimize the boundary effects on the flow development. A constant velocity profile $U_{\infty}$ was specified at the domain entrance. Uniform horizontal velocity component was used at the domain outlet $(\partial u / \partial y=0)$. Pressure value is set to zero for one node at the outlet. All velocity components are set to zero at the walls $(u=v=0)$.

Unstructured triangular and structured nonuniform rectangular grids are shown in Figure 5. 


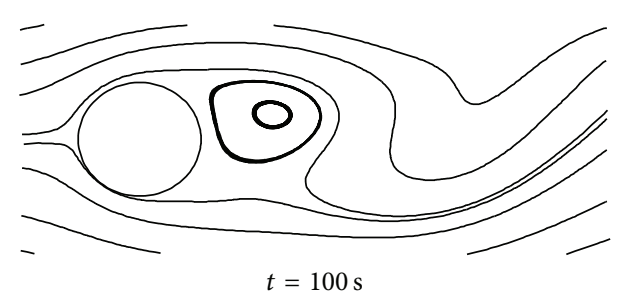

(a)

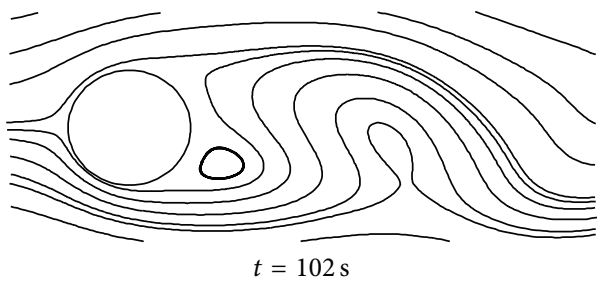

(c)



$t=104 \mathrm{~s}$

(e)

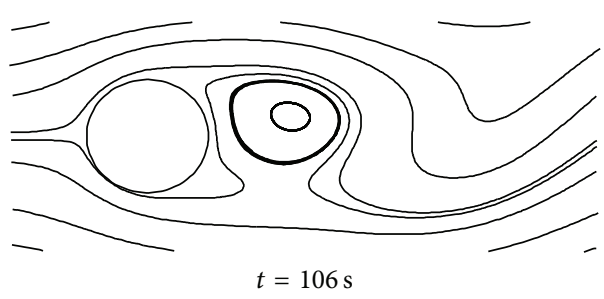

(g)

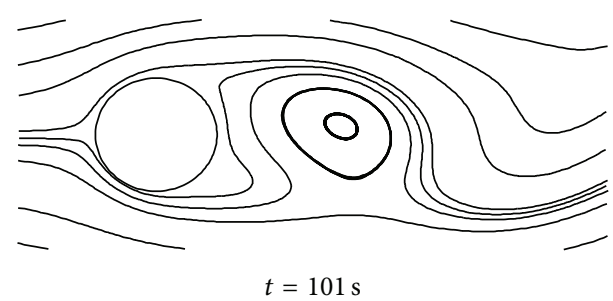

(b)

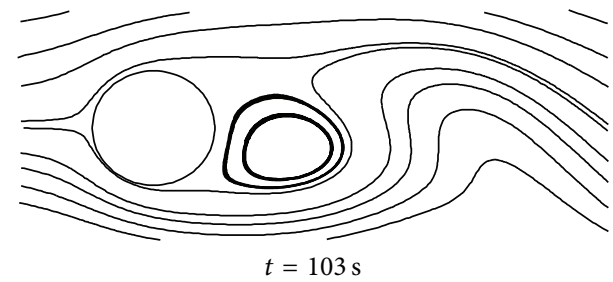

(d)

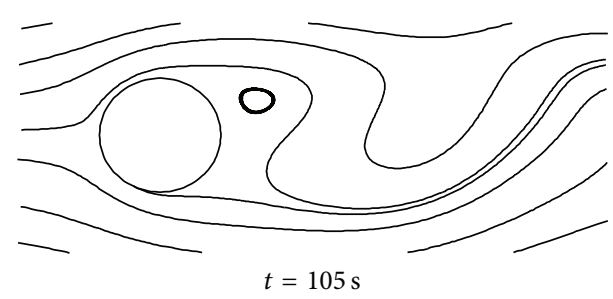

(f)

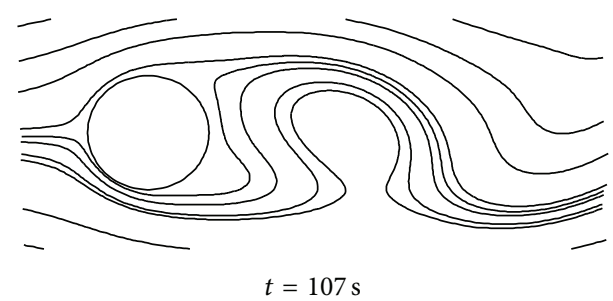

(h)

FIgURE 9: Time-dependent streamline contours for $\mathrm{Re}=100$.

\section{Results and Discussion}

For every Reynolds number, iterations are continued until both the maximum residuals of mass and momentum equations are less than $10^{-5}$ in the computational domain. Figure 6 shows the streamlines contours along the domain for $\mathrm{Re}=$ 10,20, and 40. At these Reynolds numbers, the flow is laminar and steady. These contours are the same as those of structured mesh problem. The mesh is nonsymmetrical and the lengths of recirculation flow for both sides of the geometry are different.

In all cases, a pair of vortices develops behind the cylinder and is perfectly aligned. For the laminar flow, recirculation length behind the cylinder increases with the Reynolds number. The results in this case are also shown to be in good agreement with the exiting high Reynolds number solutions $[14,15]$. Figures 7 and 8 show the pressure coefficient as a function of the angle $\theta$ on the cylinder surface for $\mathrm{Re}=$ 20,40 . Pressure coefficient is defined as

$$
C_{p}=\frac{p}{(1 / 2) \rho u_{\infty}^{2}}
$$

Pressure coefficient is compared against that computed by a structured mesh.

Some quantitative parameters, such as the length of reattachment region, $L_{\text {sep }}$, from the rearmost point of the cylinder to the end of the wake field (where horizontal velocity component direction changes) and separation angle $\theta_{\text {sep }}$ as well as the results from other researchers [9], are listed in Table 1. All these flow parameters have good agreement with the results of previous studies for all three Reynolds numbers studied. 


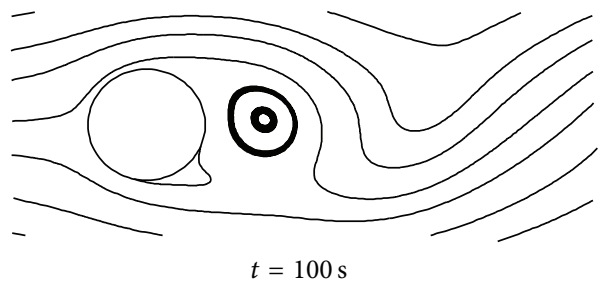

(a)

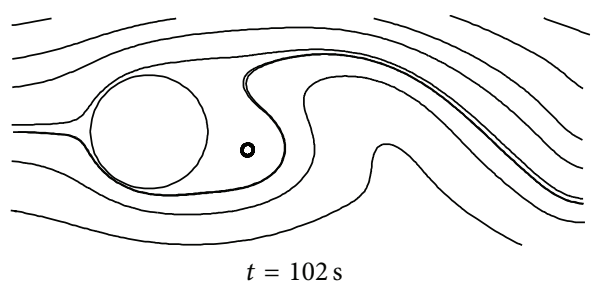

(c)

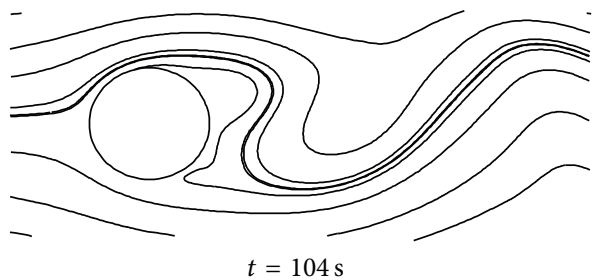

(e)

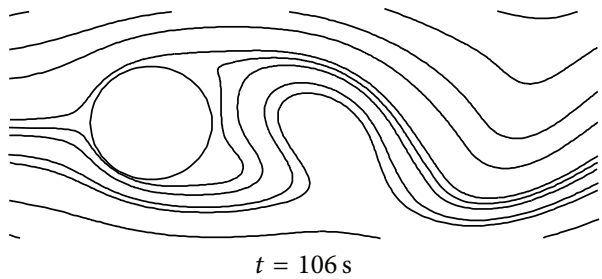

(g)

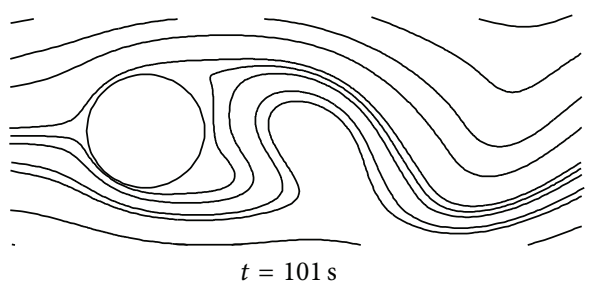

(b)

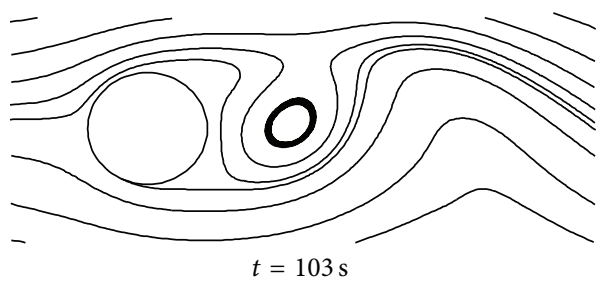

(d)

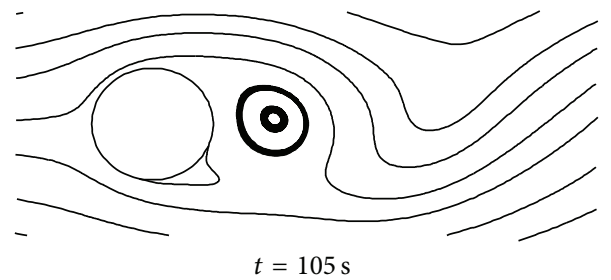

(f)

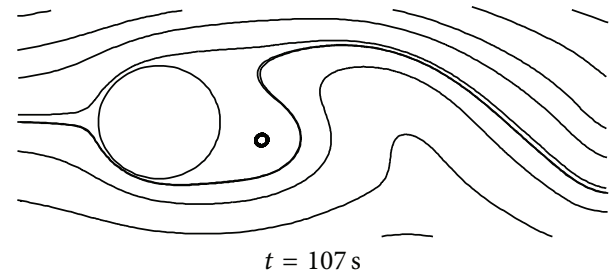

(h)

FIgURE 10: Time-dependent streamline contours for $\mathrm{Re}=200$.

At moderate Reynolds number, the flow is laminar and unsteady. In the current study, additional numerical simulations are presented for a series of extra Reynolds numbers above 100 with unsteady flow pattern. A time marching approach is performed to simulate the unsteady flow at every time step. Figures 9 and 10 show the time-dependent streamline contour for both $\mathrm{Re}=100$ and $\mathrm{Re}=200$. The periodicity of unsteady flow field has been displayed in both contour plots.

Flow in the downstream region for $\mathrm{Re}=100$ was shown to oscillate and the pattern of streamline contours repeated every 6 seconds. This period was shown to be about 5 seconds for $\operatorname{Re}=200$.

The experimental Strouhal number $(S=D / \tau U)$ for $\operatorname{Re}=$ 100,200 can be derived from [13], where $\tau$ is the period of vortex shedding. The Strouhal number is 0.165 for $R e=100$, and $\tau$ is $6.06 \mathrm{~s}$ [13]. Figure 11 shows a collection of data on the Strouhal number versus Reynolds number. It is evident that the current results agree well with the average of data shown in the figure. Figure 12 displays the time evolution of stream function value on the cylinder surface for the Reynolds numbers $\operatorname{Re}=100,200$.

\section{Conclusion}

An accurate vertex centered control volume framework based on tetrahedral element was implemented to solve NavierStokes equations in primitive form in 2D flow over a circular cylinder. In this method, fully coupled Rhie and Chow algorithm was used to suppress the probable checkerboard problem in a collocated grid system. Based on this scheme, momentum interpolation of velocity is used in conservation of mass equation and a new linear approach is implemented in momentum equation. Main advantage of the present numerical method is the fact that values are calculated at the nodal points. Therefore, boundary conditions are applied on element faces and nodes. In addition, this combination leads to simplicity and fast convergence. In this study, an unstructured tetrahedral grid has been used to create a fine mesh near the cylinder, and the results have been compared 


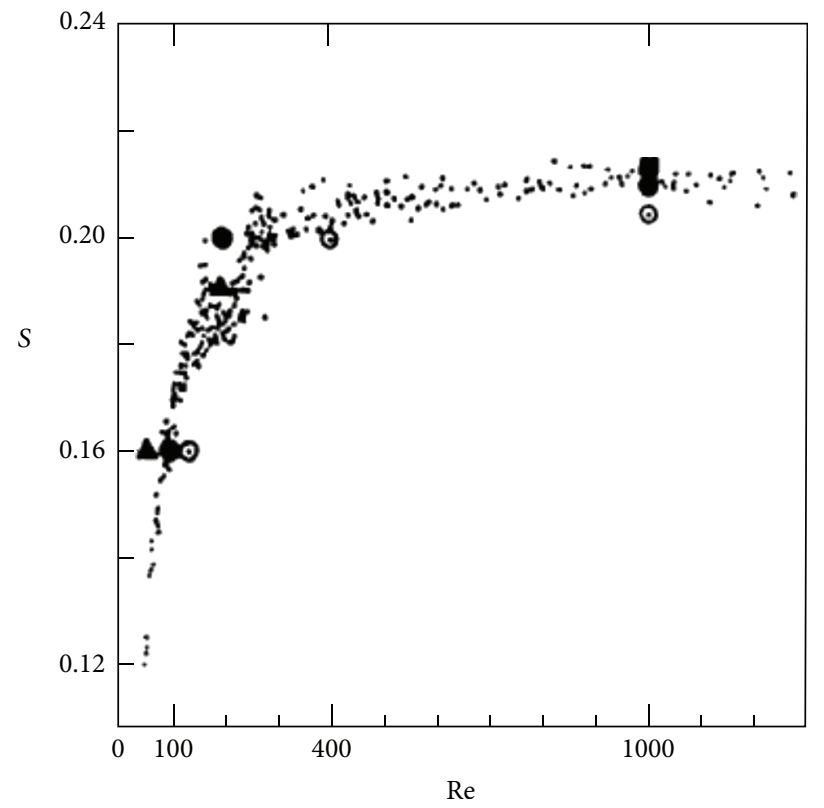

FIgURE 11: The Strouhal number versus Reynolds number [13].

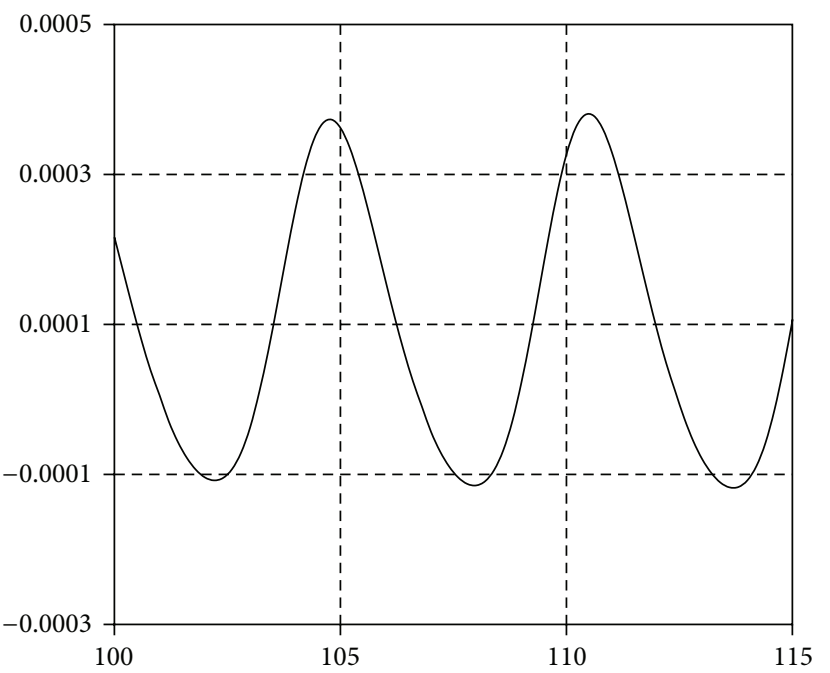

(a)

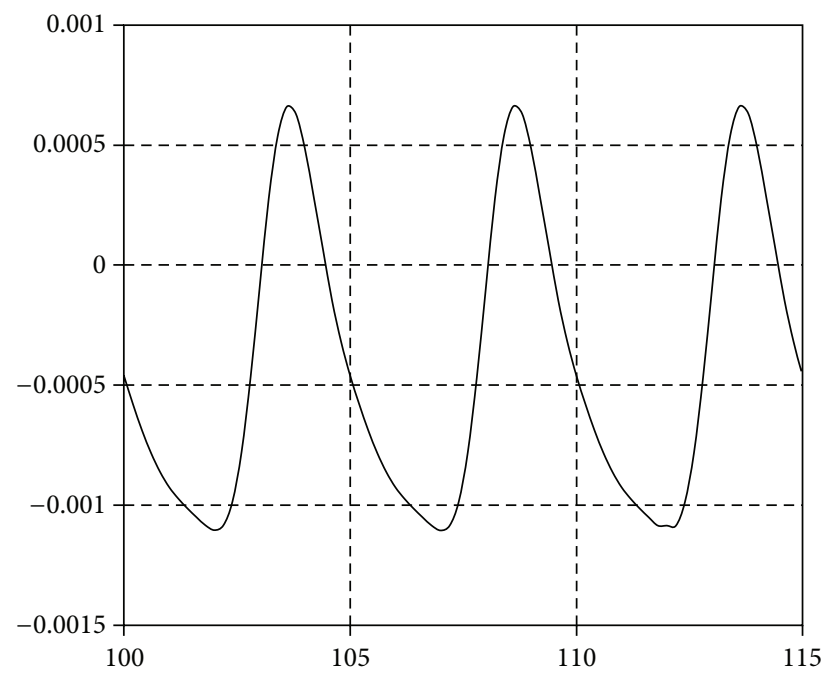

(b)

FIGURE 12: Stream function value on the surface of the cylinder for $\operatorname{Re}=100$ (a), 200 (b).

with the experimental values. In addition, this method is implemented to solve the same problem with a nonuniform quadrilateral grid and the results of both simulations have been compared.

Different flow simulations were performed at various Reynolds numbers. Results indicate that the suggested numerical scheme is proved to work for Reynolds numbers up to 200 for structured and unstructured grids. The robustness and accuracy of the present solution are proved by the comparison of pressure distribution over the cylinder for low Reynolds number. The values of structured and unstructured grids are similar. Furthermore, for the medium Reynolds number, unsteady flow has been reviewed to find the Strouhal number. The Strouhal numbers are the same as experimental data. This means that proposed collocated grid on unstructured grid is comparable with other solutions in efficiency, simplicity, and accuracy and could be used in complicated geometry.

Extending the proposed formulation and numerical schemes to three-dimensional problems may be considered as a future study.

\section{References}

[1] M. S. Bloor, "The transition to turbulence in the wake of a circular cylinder," Journal of Fluid Mechanics, vol. 19, pp. 290304, 1964. 
[2] A. Roshko, "On the development of turbulent wakes from vortex streets," Report 1191, NACA, 1954.

[3] D. Tritton, "Experiements on the flow past a circular cylinder at low Reynolds numbers," Journal of Fluid Mechanics, vol. 6, pp. 547-567, 1959.

[4] M. Nishioka and H. Sato, "Measurements of velocity distributions in the wake of a circular cylinder at low Reynolds numbers," Journal of Fluid Mechanics, vol. 65, pp. 12-1974, 1974.

[5] M. Braza, P. Chassaing, and H. H. Minh, "Prediction of largescale transition features in the wake of a circular cylinder," Physics of Fluids A, vol. 2, no. 8, pp. 1461-1471, 1990.

[6] R. Franke, W. Rodi, and B. Schönung, "Numerical calculation of laminar vortex-shedding flow past cylinders," Journal of Wind Engineering and Industrial Aerodynamics, vol. 35, no. 1-3, pp. 237-257, 1990.

[7] X. Zhang, S. Ni, and G. He, "A pressure-correction method and its applications on an unstructured Chimera grid," Computers \& Fluids, vol. 37, no. 8, pp. 993-1010, 2008.

[8] D. A. Lysenko, "Unstructured meshes in unsteady CFD applications," in West-East High Speed Flow Field Conference, pp. 19-22, Moscow, Russia, November 2007.

[9] H. Ding, C. Shu, K. S. Yeo, and D. Xu, "Simulation of incompressible viscous flows past a circular cylinder by hybrid FD scheme and meshless least square-based finite difference method," Computer Methods in Applied Mechanics and Engineering, vol. 193, no. 9-11, pp. 727-744, 2004.

[10] C. M. Rhie and W. L. Chow, "Numerical study of the turbulent flow past an airfoil with trailing edge separation," AIAA Journal, vol. 21, no. 11, pp. 1525-1532, 1983.

[11] H. Alisadeghi and S. M. H. Karimian, "Comparison of different solution algorithms for collocated method of MCIM to calculate steady and unsteady incompressible flows on unstructured grids," Computers \& Fluids, vol. 46, no. 1, pp. 94-100, 2011.

[12] L. Wang, Y. Bo, X. Wang, and S. Sun, "Calculation of cell face velocity of non-staggered grid system," Journal of Applied Mathematics and Mechanics, vol. 33, no. 8, pp. 991-1000, 2012.

[13] M. Braza, P. Chassaing, and H. Ha Minh, "Numerical study and physical analysis of the pressure and velocity fields in the near wake of a circular cylinder," Journal of Fluid Mechanics, vol. 165, pp. 79-130, 1986.

[14] S. Subhankar, M. Sanjay, and B. Gautam, "Numerical simulation of steady flow past a circular cylinder," in Proceedings of the 37th National and 4th International Conference on Fluid Mechanics and Fluid Power, Chennai, India, December 2010.

[15] A. L. F. Lima E Silva, A. Silveira-Neto, and J. J. R. Damasceno, "Numerical simulation of two-dimensional flows over a circular cylinder using the immersed boundary method," Journal of Computational Physics, vol. 189, no. 2, pp. 351-370, 2003. 



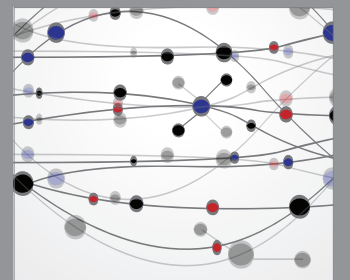

The Scientific World Journal
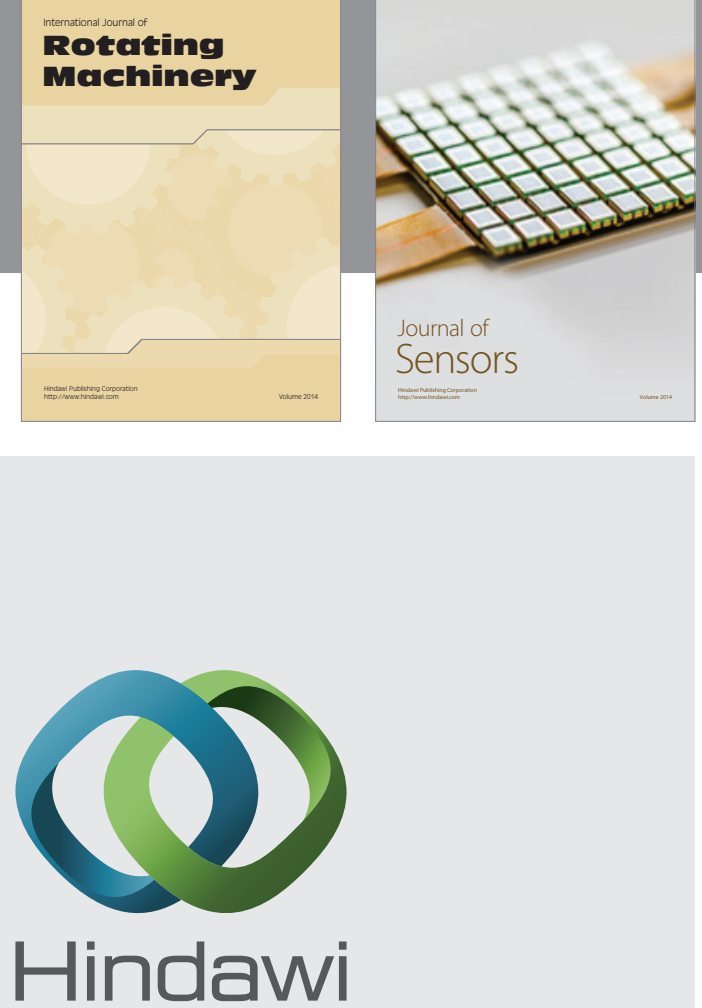

Submit your manuscripts at http://www.hindawi.com

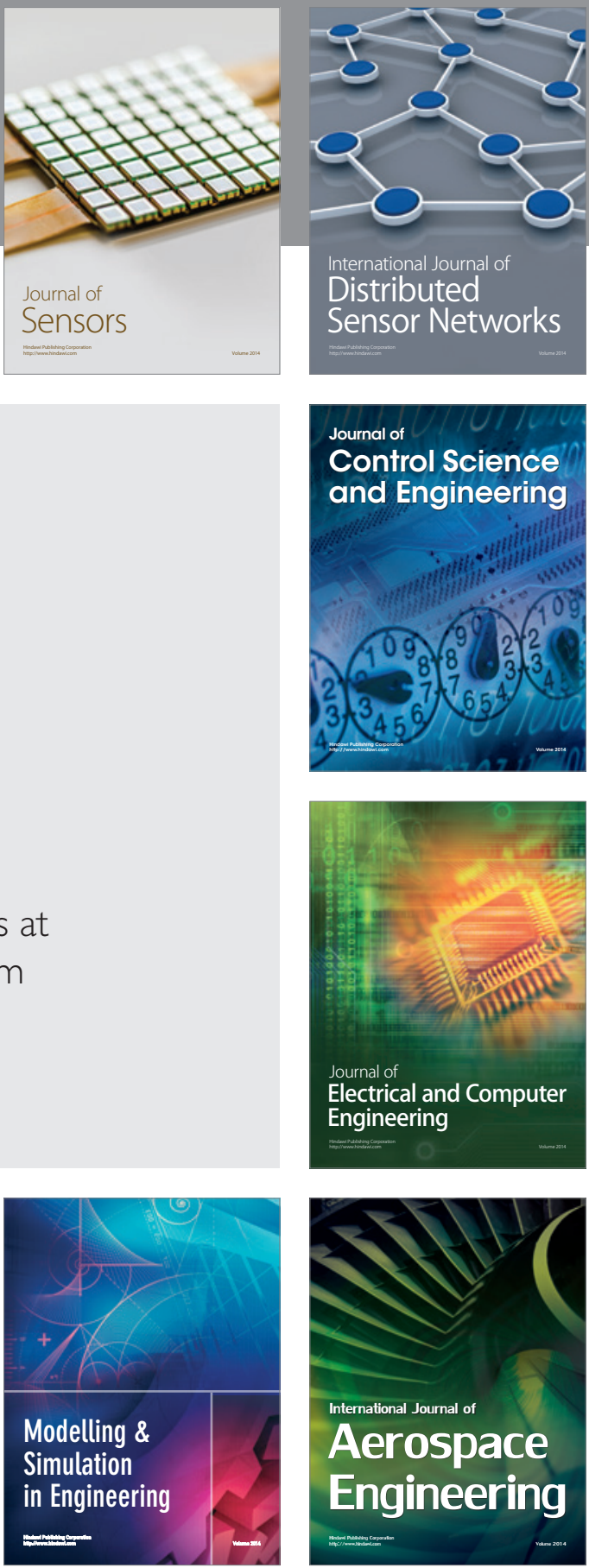

Journal of

Control Science

and Engineering
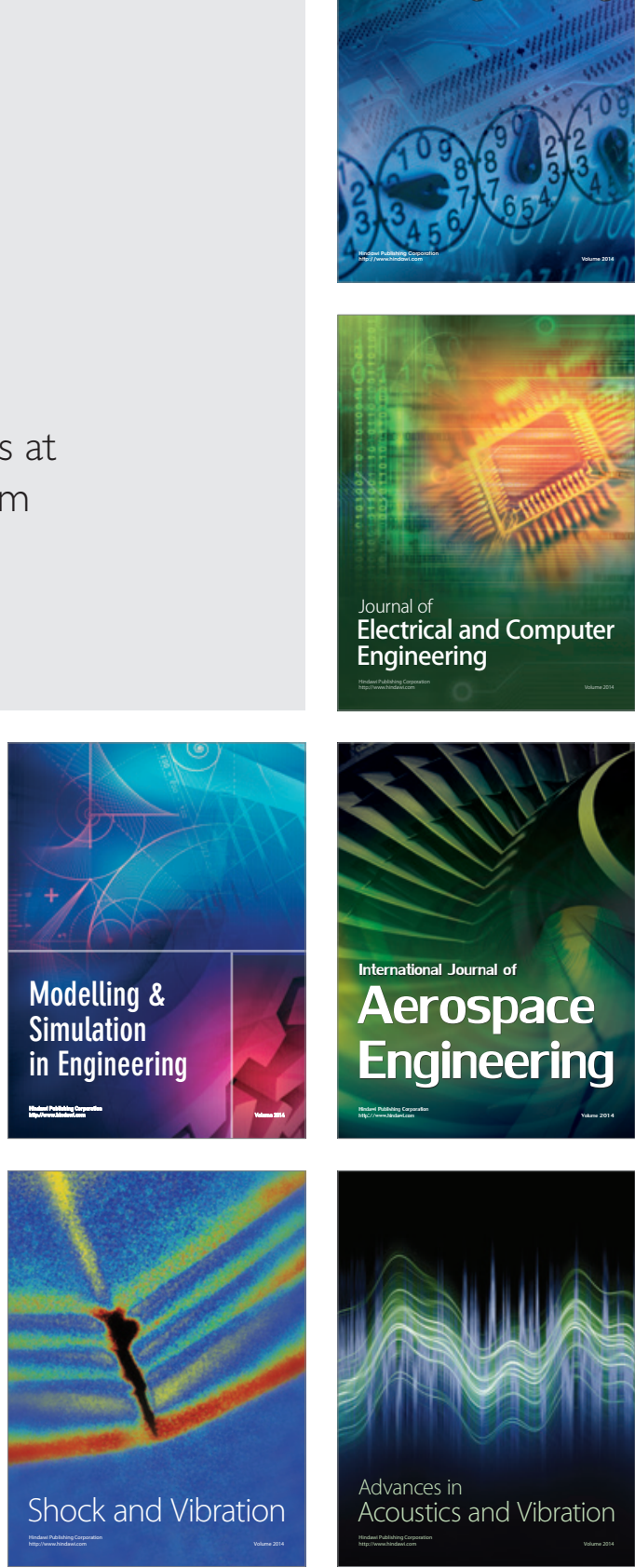